JOINT COMMISSION IV

A Proposal for an International Antarctic Observatory

Commission 9 with $40,44 \& 50$

Chairman \& Editor: P. Gillingham 


\title{
THE DEVELOPMENT OF ANTARCTIC ASTRONOMY - INTRODUCTION
}

\author{
Peter Gillingham \\ Anglo-Australian Observatory
}

This Joint Commission Meeting aimed to increase world-wide awareness of the attractions of Antarctica for a number of important classes of observational astronomy and to acquaint astronomers with the very significant amount of work already done there and the more ambitious plans now developing. Even more important, in the broad forum provided by a General Assembly, was to point out and discuss the unique promise Antarctica offers for establishing a truly international, highly productive observatory.

Papers were given under three headings: 'the attractions of Antarctica for astronomy', 'current astronomical programmes', and 'plans for the future'. The summaries are presented here in this sequence. In a half day meeting, it was unfortunately not possible to give a balanced coverage of all the fields of Antarctic astronomy; e.g. the very successful work on solar seismology was not described and some other fields were mentioned only in passing.

In considering the astronomical merits of Antarctica, it is first necessary to correct a few popular misconceptions. The area of Antarctica most favoured for astronomy - the high inland plateau is not afflicted by howling gales and heavy snowfalls, although it is, of course, very cold. Figure 1 shows contours at $3000 \mathrm{~m}$ and $4000 \mathrm{~m}$ altitude. The South Pole, where most successful astronomy has so far been accomplished, is at about $2800 \mathrm{~m}$; the highest part of the plateau is at about $4200 \mathrm{~m}$. At the top of the plateau, the wind speed is expected to average about $3 \mathrm{~m} / \mathrm{s}$ in the windiest month and the annual snow precipitation may be equivalent to as little as $10 \mathrm{~mm}$ water. Although past statistics for the dark months are probably optimistic, the

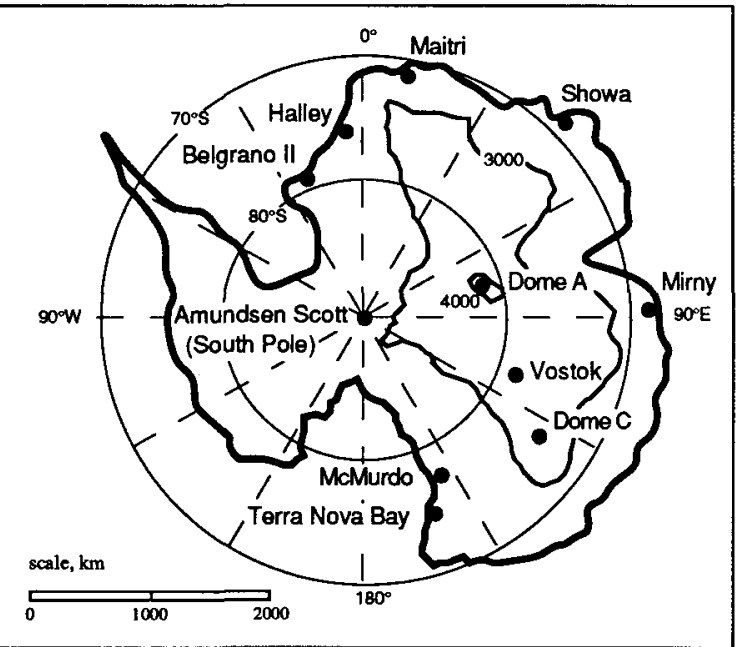

Figure 1. Sketch showing the existing and proposed bases mentioned in the following papers. proportion of clear sky should be favourable. The major attractions for astronomy result from the cold, tenuous, and extremely dry atmosphere.

The maturing of detector technologies makes the coming decade an especially propitious time to exploit the astronomical attributes of Antarctica. It is confidently expected that international collaboration, encouraged by the IAU, will increase the scientific returns to all nations which contribute to this exciting enterprise. 